\title{
Application of Network Science to Extend the AHP and QFD Methods
}

\author{
Edina Kulcsár ${ }^{1}$, István Gábor Gyurika ${ }^{1}$, Tamás Csiszér² \\ ${ }^{1}$ University of Pannonia, Institute of Material and Mechanical Engineering \\ Egyetem street 10, 8200 Veszprém, Hungary \\ kulcsar.edina@mk.uni-pannon.hu; gyurika@almos.uni-pannon.hu \\ ${ }^{2}$ Obuda University, Sándor Rejtő Faculty of Light Industry and Environmental Protection Engineering \\ Doberdó street 6, 1034, Budapest, Hungary \\ csiszer.tamas@rkk.uni-obuda.com
}

\begin{abstract}
Understanding customers' needs and developing a product which meets expectations is a multi-criteria decision problem, and requires methods for solving complex tasks. The purpose of the present study is to apply network science for prioritization of customers' needs and extend the applicability of the Analytic Hierarchy Process (AHP) and Quality Function Deployment (QFD) methods. These two methods can be used jointly in the customers' needs prioritization. The customer's needs are prioritized by using the AHP technique, and the technical requirements are ranked by using the QFD method. Although network analysis is a widely used method in several disciplines, it is not yet widespread in customer needs assessment. This study aims to fill this gap, replace and combine the AHP and QFD methods by one to facilitate the application. Preference and correlation networks are presented and evaluated in detail. By using a preference network, the importance of the customer needs is definable by calculating an in-degree or a PageRank value. In a correlation network, the effects of the technical requirements on each other can be evaluated by specifying their weights and directions.
\end{abstract}

Keywords: Network science, AHP, QFD, Pairwise comparison

\section{Introduction}

Understanding customer needs is essential to develop cost-efficient and vendible products. In today's accelerated world, companies need to respond to customer habits and needs very quickly, which requires sufficiently detailed and fast decision support methods. In this study, it is examined how network analysis can replace and extend the QFD and AHP methods to reduce application time and to make associated activities easier to do. We prove that customer requirements can be structured and analysed as a complex network. As its evidences, some possible networks, their attributes and assessment possibilities are introduced in the following sections. Then we present how network analysis can be used to assess the pairwise comparison-based questionnaire and its consistency. Finally, we evaluated the correlation relationships between technical requirements by network analysis techniques. As for IT applications, Gephi and NodeXL were used for visualization and evaluation.

\section{Literature background}

The Analytic Hierarchy Process (AHP) and Quality Function Deployment (QFD) are widespread methods for analysing customer demands. The AHP method was developed by Saaty. The AHP structures problems in a hierarchy and the evaluation is based on pairwise comparison. The pairwise comparisons are performed with a 1-9 scale and the results are stored in a symmetric matrix. From the matrix, the inconsistency of the customer evaluation and the prioritization of the alternatives are computable. The consistency can be measurable in the case of three criteria. The result is an inconsistent circle if $\mathrm{B}$ is preferable to $\mathrm{A}, \mathrm{C}$ is preferable to $\mathrm{B}$, and $\mathrm{A}$ is preferable to $\mathrm{C}$. The acceptable inconsistency limit is generally smaller than $10 \%$ [1].

QFD was developed by Yoji Akao in Japan. It is a method to help transform the customer needs into technical characteristics and collect detailed information to start product development [2]. The first step of the method can be a customer survey to identify and rank the importance of the features of a product. Characteristics are also defined, which can satisfy these customers' requirements. The connection between the requirements and the characteristics are defined in a 
relationship matrix. Positive or negative correlation between the characteristics can be assessed in another correlation matrix. A competitive assessment of our own and our competitors' current position relative to the customer importance is also part of the analysis. The outputs of the QFD are the functions to be developed. The last parts of the assessment of the first house of QFD are to determine importance ratings to the functions and, according to results, specify their target values[3].

These two methods were applied together many times for different purposes. QFD and AHP were integrated to select suppliers in the automotive industry. QFD was used to translate the requirements into multiple evaluating factors, and AHP was applied to determine the importance of these evaluating factors [4]. In another application, QFD provided the importance weights of the criteria, and the suppliers were evaluated and compared by AHP [5]. An iterative AHP-QFDAHP approach for brainstorming was also implemented when a new concept was developed. These two methods were used for creative thinking [6]. An integrated AHP and QFD approach was proposed in another application to select the most appropriate abatement technology. Both methods were used for ranking the technologies [7]. To determine the preferences of the physicians in prescribing the drugs in Bangladesh, AHP was used to derive weights to the criteria and QFD was used to rank the technical aspects [8]. It can be seen from the literature, that these two methods are well applicable together widely [9]. However, their use requires throughout knowledge of the details of the methods, which can cause more complicated, longer application time.

The foundations of network research were laid by a Hungarian researcher, Albert-László Barabási, who has been actively involved in its application. Network science deals with complex networks such as telecommunication networks, social networks, biological networks, computer networks, etc., represented by nodes and their links [10]. Network analysis was already applied as multidimensional customer-product network (MCPN) to model customer preferences and support design decisions. Two layers were created, one was the customer, and the other was the product where the connections present the consideration, purchase, product associations, etc. [11]. A PageRank algorithm was also used to prioritize requirements, and it proved to be suitable for prioritization of customer needs [12][13]. However, these applications did not focus on the replacing and extending the AHP and QFD methods. As a result of the literature review, there are many articles close to the topic, but it has been stated that the applicability of network analysis has not been examined in detail in the applications of the customer demand analysis.

\section{Preference and consistency evaluation}

A possible way for calculating the importance of product functions and evaluating the consistency of the answers by network analysis is shown in this section. The ranking of the importance of the customer needs is part of the QFD method as its first step, for which the AHP method can be applied. For both methods, the customer's opinion is usually measured by a questionnaire using paired comparisons. A pairwise comparison-based questionnaire can also be assessed by using network analysis.

\subsection{Preference network}

In the preference network studied below, the vertices represent the customer needs, and the directed edges represent the preferences. The weight of the preference can also be asked in the questionnaire and assessed in the network as in the AHP method. Therefore, the preference network is directed and weighted where there are connections among all the vertices, which results in a complete graph. The following network analysis attributes were examined considering their applicability:

- In-Degree: In a directed graph, in-degree $\left(k_{i}^{i n}\right)$ represents the number of other vertices that point to the selected vertex [10];

- PageRank: PageRank is an algorithm to determine the importance of a vertex in a network. The algorithm is based on how many connections the vertex has and how important they are [14].

In the example, pairwise comparison was used to assess 19 functions as $F_{i}$, the results were generated randomly, and every function pair was assessed twice. The weight of the preference was also given in the evaluation: 1 as more important and 2 as much more important. 
The results of the calculations of in-degree and PageRank value can be seen in Fig.1. The colour represents the indegree of the vertices in both cases (the darker the vertex, the higher the value of the in-degree), and the diameter of the vertices shows the magnitude of the in-degree on the a) graph and the magnitude of the PageRank on the b) graph (the bigger the vertex, the bigger the magnitude of the indicator). The weights of the edges were summed if the direction of preferences were the same at the same pairing. In case of an inconsistent answer, both edges are shown and assessed in both directions, which can be seen in Fig. 2. The PageRank values were calculated by taking into account the weights of the edges too. $F_{1}, F_{5}, F_{7}$ and $F_{19}$ have the greatest importance in the graph according to the in-degree and the PageRank values.

Based on the results, network research proves to be suitable for determining the weight of preference, as it can be determined by two different network indicators. The PageRank algorithm takes into account the importance of the other nodes; therefore, the network analysis offers a different approach instead of the prioritization methods in the AHP and QFD methods.

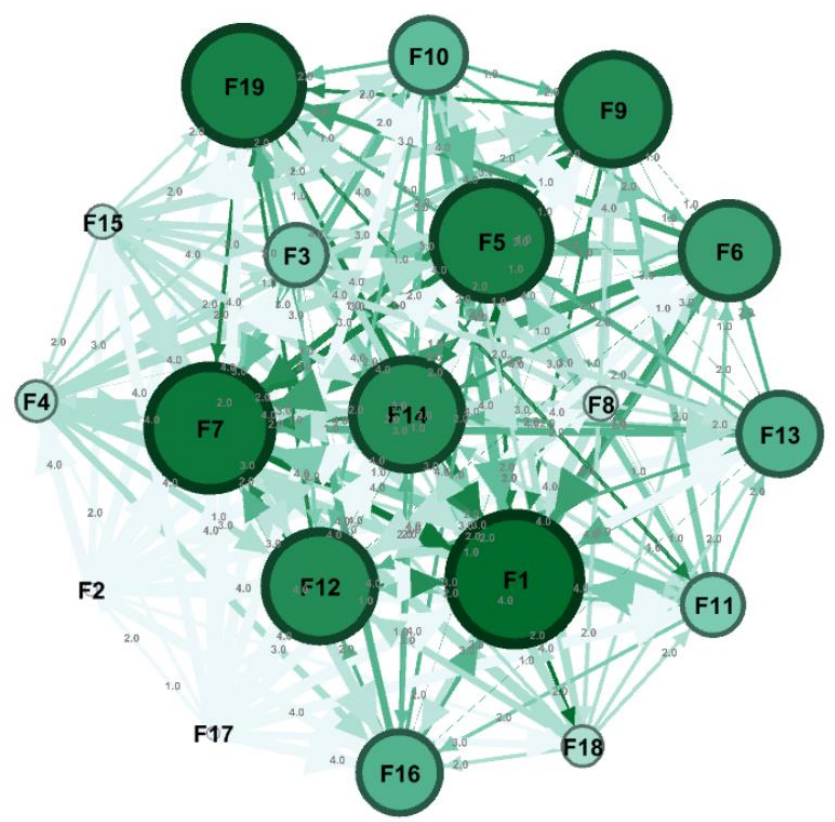

a)

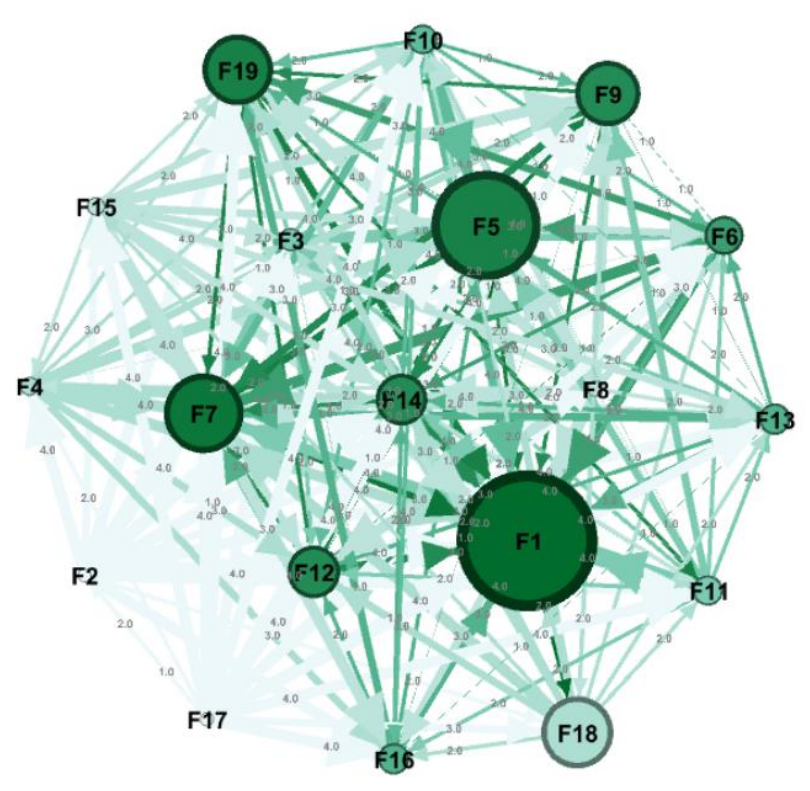

b)

Fig. 1: a) preference networks to visualize the in-degree of the vertices and b) PageRank of the vertices. The diameter of the vertices reflects the values of in-degree and PageRank (the higher the value of the indicator is, the bigger the diameter of the vertex is). The colours of the vertices show the magnitude of the in-degree in both cases. (Visualization was created in Gephi.) 


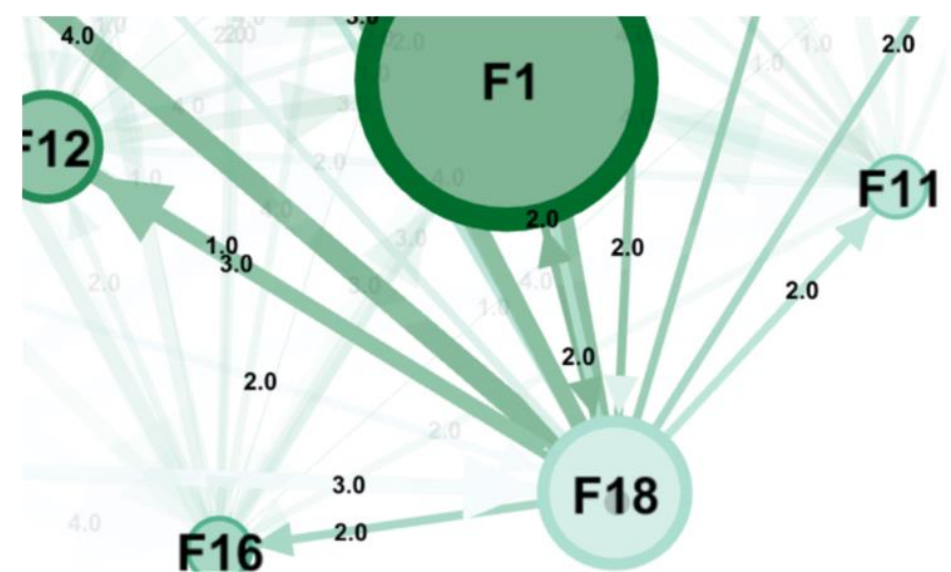

Fig. 2: Types of the preferences and their weights. Weights of the preferences are summed if the answers were consistent in case of the same pairing. When the answers are inconsistent, the edges are reciprocated and the weights are evaluated in both directions. The colour intensity shows the magnitude of the weight. (Visualization was created in Gephi.).

\subsection{Consistency evaluation}

The consistency is generally measurable in case of three evaluation factors. By using network analysis, consistency evaluation is applicable even for only two factors, if the comparison is provided twice. In the graph, the reciprocated edges show the inconsistent comparisons as it can be seen in Fig. 2.

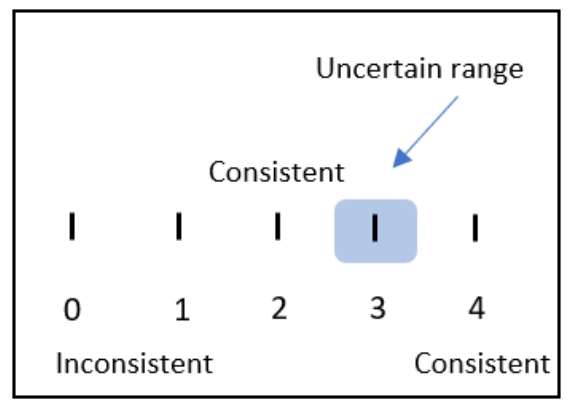

Fig. 3: The possible sum of weights, taking into account the direction of the preferences.

If we examine the uncertainty of the answers in the example while taking into account the direction of the preference, we get the following cases (presented in Fig. 3), where the minus (-) and plus (+) signs indicate the different directions. In case of opposite directions, one of the edges gets a positive sign if it has a higher weight.

- Inconsistent answer: The directions of the preferences are contrary, and the weights are -2 and 2 , or -1 and 1 , when the summed value is 0 . The other case the weights can be -1 and 2 , when the sum of the weights is 1 ;

- Consistent answer: The weights are 2 or 1 twice in the same direction; therefore, the summed values are 4 or 2;

- Uncertain answer with the same direction of the preference: The weights are 1 and 2 in the same direction, and the sum of the weights is 3 .

The number of the reciprocated edges and the inconsistent weights can be easily calculated in NodeXL. The limit of the consistency without taking into account the weight of the responses in a graph can be calculated with the following formula:

$$
C_{l}=1-\left(z_{i} / z_{\max }\right) \cdot 100 \%
$$


where $C_{l}$ means the limit of the consistency, $z_{i}$ represents the number of inconsistent answers and $z_{\max }$ is the number of possible inconsistent answers, which is the half of the number of comparisons. The inconsistent answers can be excluded from the evaluation by slicing the network.

\section{Correlation between the technical requirements}

In this section, a possible evaluation of the correlations between product technical requirements by network analysis is demonstrated. The method of the roof matrix of QFD was used for calculating the correlation.

The correlation between two technical requirements can be negative, positive or neither. The correlation network is directed, where the direction represents the effect. The weight of the correlation can also be determined. In our example, the weight could take a value from -3 to 3 , where 0 means no correlation, and the two extremes values represent the strongest negative or positive correlations, respectively. 21 technical requirements, denoted by $M_{i}$, were identified and assessed. The correlation relationships were generated randomly. In Fig. 4, the colour intensity represents the value of the in-degree and the diameter shows the value of the out-degree.

In a directed graph, out-degree $\left(k_{i}^{\text {out }}\right)$ represents the number of connections that point from the vertex to other vertices [10]. The magnitude of the effect can be specified by the weighted out-degree and the weighted in-degree. Based on the value of the out-degree and in-degree of a vertex, the following cases can be distinguished.

- Negative out-degree: Changing the technical requirement has a negative effect on many other technical requirements $\left(M_{1}\right.$ in the example);

- Positive out-degree: Changing the technical requirement has a positive effect on many other technical requirements ( $M_{2}$ in the example);

- Negative in-degree: Changing several technical requirements have negative effects on it. This is especially worth looking at when the examined technical requirement influences an important customer demand $\left(M_{9}\right.$ in the example);

- Very positive in-degree: Changing several technical requirements have positive effects on it ( $M_{21}$ in the example);

- Biggest impact in the network: The vertex has the biggest out-degree in the network $\left(M_{1}\right.$ in the example);

- Isolated vertex: It has no correlation to any other vertex. This technical requirement can be changed independently $\left(M_{12}\right.$ in the example);

- Null in-degree: It is not affected by any other technical requirement $\left(M_{2}, M_{3}\right.$ in the example);

- Null out-degree: It has no effect on any other technical requirement. This technical requirement can also be changed independently $\left(M_{4-14}\right.$ and $\left.M_{16-21}\right)$.

By examining these properties, the decision as to which function to change can be supported. If a technical requirement is changing, it may influence negatively the technical requirement that is considered the most important from the customer's perspective. The chain of the impact along different paths can also be determined to examine indirect effects. Network analysis proves to be applicable to evaluate and visualize the correlation relationships between the technical requirements. 


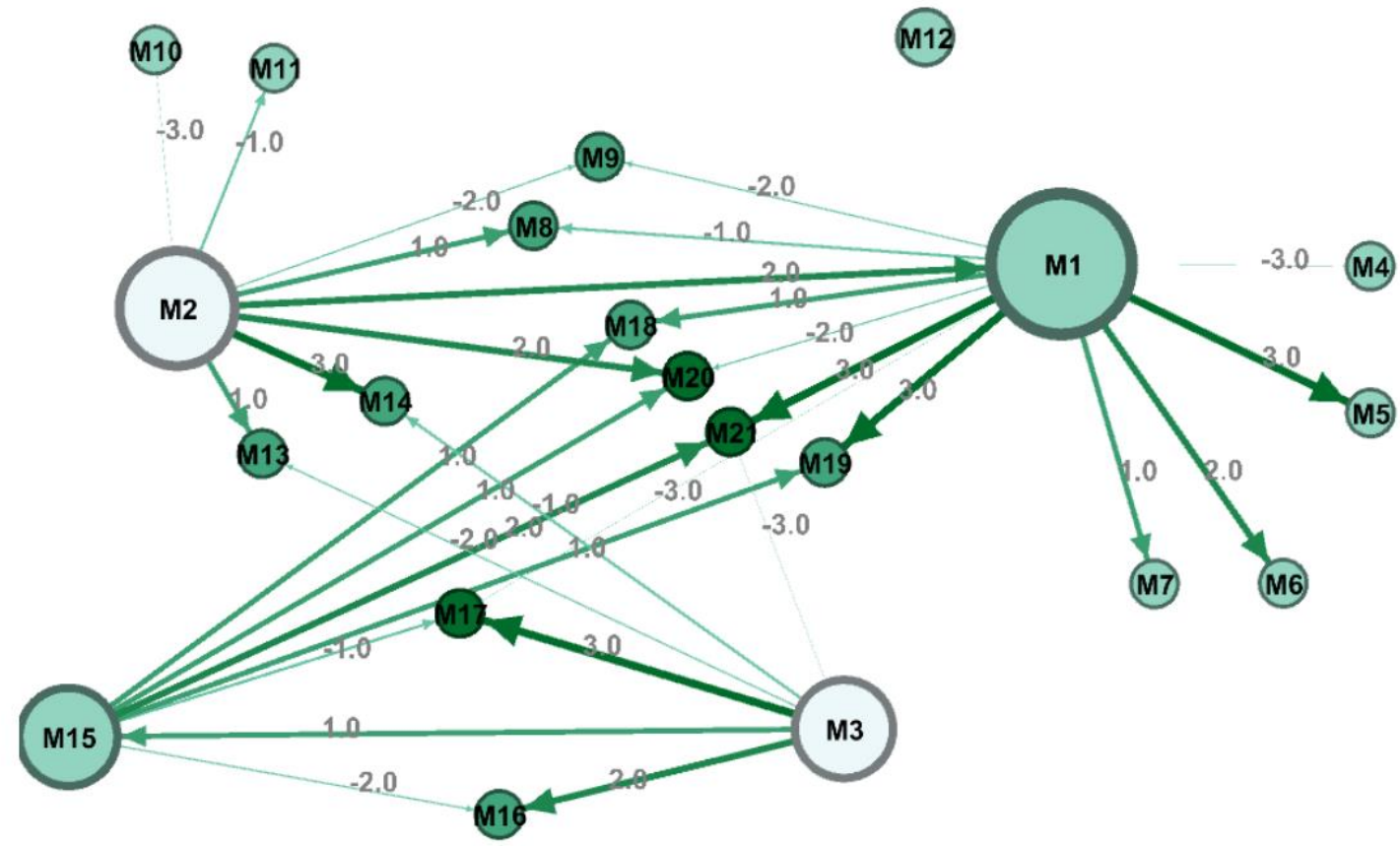

Fig. 4: Correlation network of technical requirements. The diameter of the vertex shows the magnitude of the out-degree and the colour of the vertices shows the magnitude of the in-degree. The Bigger the vertex is, the higher the value of the out-degree, the darker the vertex is, the higher the value of the in-degree. The weights of the edges represent the significance of the effect. (Visualization was created in Gephi.).

\section{Conclusion}

In the previous sections, it was examined how network analysis can be applied to replace and extend the AHP and QFD methods. The customer preferences and the consistency of their answers were evaluated by using network analysis. The in-degree and the PageRank of a vertex were calculated to determine the importance of the customer needs. In the case of pairwise comparison, the weight of the preference can also be specified, without limitation. Consistency is generally measurable in the case of three criteria. By using network analysis, consistency evaluation can be carried out for two criteria too. The presented applications are not only suitable for prioritizing customer needs, but also for analysing any other type of preferential relationships.

The application of a correlation network can support the decision on which technical requirement should be modified. By calculating the out-degree and in-degree, interactions in the network can be easily defined as well. The most influential nodes can be identified, as well as the correlation relationships of the technical requirements, which are influencing the factor that is important for the customer. Network analysis also helps to visualize the relationships of the technical requirements and customer needs. The visualization facilitates the understanding and evaluation of the relationships and correlations.

Another area of research can be the development and replacement of other steps of QFD using network analysis, as well as the development of the consistency evaluation for the $n>3$ criteria. It is worth considering the possibility of using network analysis for replacing and extending additional decision support methods.

\section{References}

[1] Saaty, Thomas L. "Decision making - the analytic hierarchy and network processes (AHP/ANP)." Journal of systems science and systems engineering 13.1, 2004, pp. 1-35. 
[2] Prasad, Biren. "Review of QFD and related deployment techniques." Journal of manufacturing Systems 17.3, 1998, pp. 221-234.

[3] Erdil, Nadiye Ozlem, and Omid M. Arani. "Quality function deployment: more than a design tool." International Journal of Quality and Service Sciences, 2019.

[4] Rajesh, G., and P. Malliga. "Supplier selection based on AHP QFD methodology." Procedia Engineering 64, 2013, pp. 1283-1292.

[5] Khan, Sharfuddin Ahmed, Fikri Dweiri, and Vipul Jain. "Integrating analytical hierarchy process and quality function deployment in automotive supplier selection." International Journal of Business Excellence 9.2, 2016, pp. $156-177$

[6] Ucler, Caglar. "Brainstorming the cryoplane layout by using the iterative AHP-QFD-AHP approach.” Aviation 21.2, 2017, pp. 55-63.

[7] Gupta, Alok Kumar, and Bharat A. Modi. "Selection of sustainable technology for VOC abatement in an industry: an integrated AHP-QFD approach." Journal of The Institution of Engineers (India): Series A 99.3, 2018, pp. 565578.

[8] Elahi, F., Ahmed, S., Haque, M., and Chowdhury, N. "An Empirical Investigation into Physician Preferences in Drug Prescription: An Integrated Methodology of AHP and QFD." International Journal of Marketing Studies 8.5, 2016, pp. 81-89.

[9] Sivasamy, K., Arumugam, C., Devadasan, S. R., Murugesh, R., and Thilak, V. M. M. "Advanced models of quality function deployment: a literature review." Quality \& quantity 50.3, 2016, pp. 1399-1414.

[10] Barabási, Albert-László. Network science. Cambridge university press, 2016.

[11] Wang, M., Chen, W., Huang, Y., Contractor, N. S., and Fu, Y. "Modelling customer preferences using multidimensional network analysis in engineering design.” Design Science 2. 2016.

[12] Abbas, M., Inayat, I., Jan, N., Saadatmand, M., Enoiu, E. P., and Sundmark, D. "MBRP: Model-Based Requirements Prioritization Using PageRank Algorithm." 2019 26th Asia-Pacific Software Engineering Conference (APSEC). IEEE, 2019.

[13] Gu, J., Liu, C., Fu, S., Mao, E., and Pang, C. "A priority index based method for identifying influential design factors in product service-oriented designs." Journal of Intelligent \& Fuzzy Systems Preprint, 2020, pp. 1-18.

[14] Page, L., Brin, S., Motwani, R., and Winograd, T. "The pagerank citation ranking: Bringing order to the web." Stanford InfoLab, 1999. 